\title{
Blind source separation of multichannel neuromagnetic responses
}

\author{
Akaysha C. Tang ${ }^{a, b, c, *}$, Barak A. Pearlmutter ${ }^{b, c}$, Michael Zibulevsky ${ }^{\mathbf{b}}$, \\ Scott A. Carter ${ }^{\mathrm{a}}$ \\ ${ }^{a}$ Department of Psychology, University of New Mexico, RM 162, Logan Hall, Albuquerque, NM 87131, USA \\ ${ }^{\mathrm{b}}$ Department of Computer Science, University of New Mexico, FEC 157, Albuquerque, NM 87131, USA \\ ${ }^{\mathrm{c}}$ Department of Neurosciences, University of New Mexico, NM 87131, USA
}

Accepted 13 January 2000

\begin{abstract}
Magnetoencephalography (MEG) is a functional brain imaging technique with millisecond temporal resolution and millimeter spatial sensitivity. The high temporal resolution of MEG compared to fMRI and PET (milliseconds vs. seconds and tens of seconds) makes it ideal for measuring the precise time of neuronal responses, thereby offering a powerful tool for studying temporal dynamics. We applied blind-source separation (BSS) to continuous 122-channel human magnetoencephalographic data from two subjects and five tasks. We demonstrate that without using any domain-specific knowledge and without making the common assumption of single- or multiple-current dipole sources, BSS is capable of separating non-neuronal noise sources from neuronal responses and also of separating neuronal responses from different sensory modalities, and from different processing stages within a given modality. (C) 2000 Elsevier Science B.V. All rights reserved.
\end{abstract}

Keywords: Functional brain imaging; Independent components analysis; Magnetoencephalography; Blind source separation

\section{Introduction}

The brain's neuromagnetic signals are small compared to the noise (signal strength: $100 \mathrm{fT}$; sensor noise: $10 \mathrm{fT}$; noise in a shielded environment from magnetized lung

\footnotetext{
* Correspondence address. Department of Psychology, University of New Mexico, RM. 162, Logan Hall, Albuquerque, NM 87131, USA. Tel.: + 1-505-277-4025; fax: + 1-505-277-1394.
} 
contaminants: $10^{6} \mathrm{fT}$; from abdominal currents: $10^{5} \mathrm{fT}$; from heart and eyeblinks: $10^{4} \mathrm{fT}$; from epileptic and spontaneous activity: $10^{3} \mathrm{fT}$ [8]) and therefore neuronal responses evoked in cognitive tasks are usually averaged across many trials of stimulation. Recently, a number of blind source separation (BSS) algorithms have successfully separated mixtures in other domains [5,9] without any special knowledge of the nature of the mixed signals or the mixing process. Most relevant to multichannel brain imaging data, Bell-Sejnowski Infomax [1] and Fast ICA [6] have successfully isolated and removed major artifacts from EEG and MEG data $[7,11]$.

In MEG, magnetic activity from different neuronal populations is observed by sensors arranged around the subject's head. Each sensor responds to a linear mixture of the signals emitted by multiple sources. Responses from different neuronal populations will be referred to as source responses and the neuronal populations that give rise to these responses will be referred to as neuronal sources or simply sources. These neuronal sources often, but not necessarily, consist of a spatially contiguous population of neurons. BSS separates the measured sensor signals into components which are as independent as possible, each with its own field map. Using spatial and temporal constraints, we will establish a correspondence between the BSS components and noise sources, and between BSS components and various neuronal sources.

\section{Methods}

Two volunteer subjects performed a total of five visual reaction time tasks which differed in the amount of processing required for target determination. In all but one task, extraction of both object identity and object location was required. Each trial consisted of a pair of colored abstract block compositions, presented symmetrically and simultaneously on the left and right halves of a screen. Subjects were instructed to respond as quickly and accurately as possible with a left- or right-hand mouse button press when a target stimulus was presented to the left or right side of the screen, respectively.

Neuromagnetic signals were recorded continuously throughout the experiment at $300 \mathrm{~Hz}$ using a whole-head 122-channel direct-current superconducting quantum interference device (DC-SQUID) neuromagnetometer (Neuromag, Helsinki, Finland.) A band-pass filter $(0.1-100 \mathrm{~Hz})$ was applied. This high dimensionality and large volume of data of continuous MEG data imposes a severe computational burden. After experimenting with the Infomax [1], cICA [9] and Fast ICA [6] algorithms, we settled on a variant of the SOBI algorithm [2], which gave sufficient accuracy (in the sense of properly separating out known and previously unknown sources of noise) at a reasonable computational cost.

\section{Results}

The BSS process separates out as many components as there are sensors, in this case 122 components. Some of these correspond to responses of neuronal populations 


\section{MEG Image}

Field Map

(a) Subject \#1 component 1 (60Hz contamination)
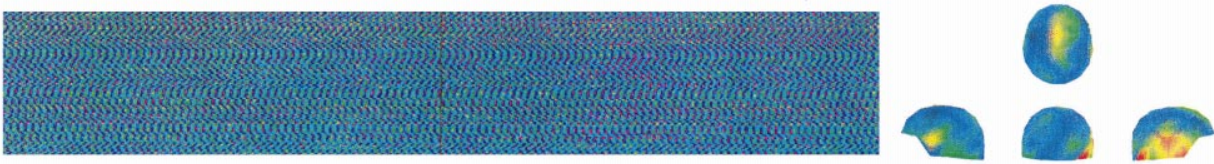

(b) Subject \#1 component 20 (slow DC drift)
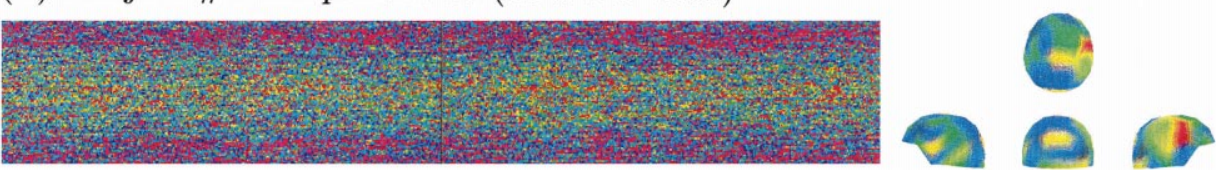

(c) Subject \#1 component 6 (eyeblinks)
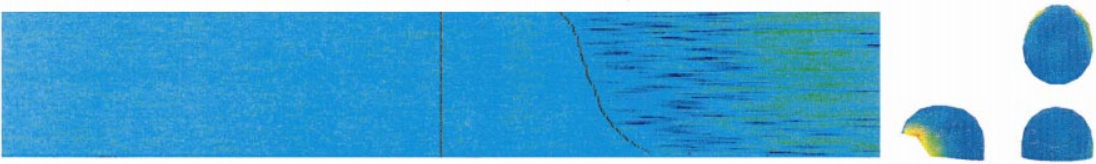

(d) Subject \#1 component 27 (occipital)
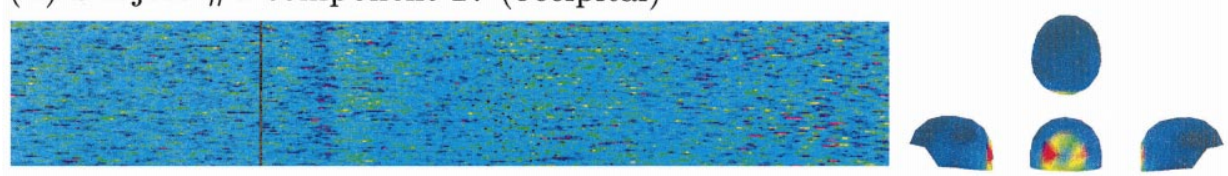

(e) Subject \#1 component 14 (occipital-parietal)
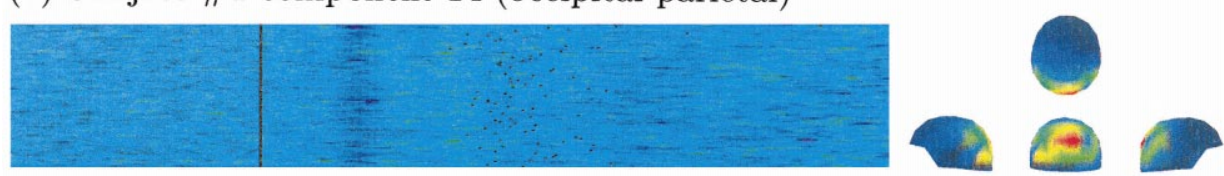

(f) Subject \#1 component 7 (right somatosensory)
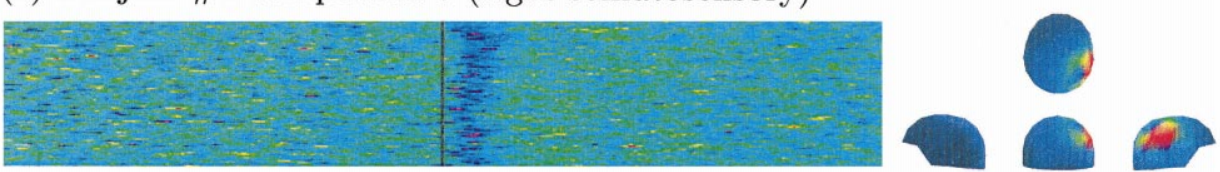

(g) Subject \#1 component 34 (left somatosensory)
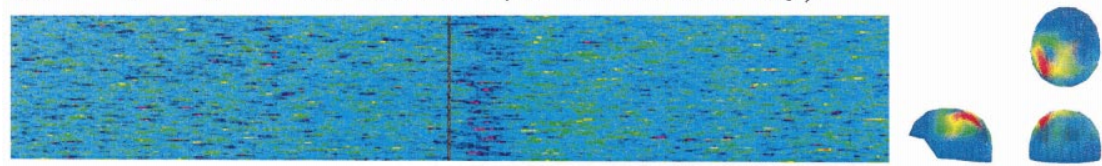

Fig. 1. Separation of noise sources and neuronal components using blind source separation. Left: $M E G$ images. Each panel contains 90 trials. Trials are ordered vertically, except for (c), which is sorted by reaction time and where the curve on the right indicates the times of button presses. Pixels are color-coded source strength. Vertical bars on the left indicate stimulus onset time. Pre- and post-stimulus durations are each 1000 ms. Right: field maps. Left sagittal, right sagittal, dorsal, and posterior views. False colors indicate the strength with which the component influences the 61 sensor pairs. MEG images and field maps of (a) a $60 \mathrm{~Hz}$ component, (b) a slow DC drift component, (c) an eyeblink component, (d) an early occipital visual component (27), (e) a later occipital-parietal visual component (14), (f) a right somatosensory component (7) and (g) a left somatosensory component (34). 
that are either task dependent or task independent; others, to various noise sources such as muscle activity due to eyeblinks, $60 \mathrm{~Hz}$ from electrical equipment, slow drift due to unknown causes, signals from dental fillings, sensor quantum jumps, and other unknown noise sources associated with random neuronal activity. Many recovered components are due to random factors not under experimental control and can be discarded. Only a subset of the separated components can be identified using their spatial and temporal properties and/or prior knowledge of the experiment.

BSS components are characterized by both MEG images and field maps. The MEG image provides temporal information and the field map provides spatial information about a particular component. When both the temporal and spatial information are consistent with anatomical and neurophysiological properties of a known neuronal population, the activity of the component is considered to reflect the activity of the known neuronal population. Confirmation and verification of component responses associated with different processing stages, e.g. early and late visual components, are provided by satisfying the temporal and spatial constraints on early and later visual processing set by our knowledge of the visual system.

Fig. 1 shows examples of known noise sources $(\mathbf{a}, \mathbf{b})$, eyeblinks $(\mathbf{c})$, visual sources (d,e), and somatosensory sources (f, g). The $60 \mathrm{~Hz}$ source contained exactly 60 cycles in a window of $1000 \mathrm{~ms}$, and had a rather distributed field map. The slow DC drifting source did not show any stimulus-locked changes but changed slowly across trials, and also had a rather distributed field map. Eyeblinks were confirmed by an independently collected EOG signal and by the field activation around the relevant muscle groups. Visual sources associated with early and later processing were established by both their response latency (see MEG images) and the occipital and occipital-parietal activation of their field maps. Somatosensory sources $(7,34)$ were identified by their button-press-locked responses, and are shown aligned by button press $(1000 \mathrm{~ms}$ preand post-button press). Their field maps are consistent with activation of the right and left somatosensory cortex. These typical noise and neuronal sources were consistently identified in all five experiments performed by both subjects.

\section{Discussion}

We applied a blind source separation algorithm to continuous 122-channel MEG data. The separated components include identifiable known noise sources and neurophysiolgically and neuroanatomically meaningful neuronal components. These neuronal components can be viewed as "cleaned" source responses, and can be fed to any existing source localization algorithm for further localization using co-registration of MEG and MRI. Since these neuronal components are already separated from many noise sources and from each other, we expect improved accuracy in future source localization, when blind source separation is coupled with well-developed source localization methods.

One fundamental difference between BSS and source localization algorithms traditionally used for MEG [4] is that BSS does not assume any prior physical source model. BSS algorithms only require temporal information from the sensor signals to 
generate an approximation of the original source signals, without making reference to or conclusions about spatial locations of the sources. As a result, BSS can deal equally well with source signals generated by a population of neurons that are best described as a point, a cylinder, a large wrinkled sheet, or neuronal populations discretely distributed. The fact that BSS does not require a physical model for its sources guarantees that there will be no separation errors arising from false or non-optimal physical source models. Consistency with a known physical model can instead be used to test for correct separation. This flexibility also permits the use of a wider and more ecological range of stimuli, which often do not generate well-defined focal activation.

The ability of the BSS algorithm to separate out various noise sources and physiologically and anatomically interpretable neuronal components arises from its use of the temporal characteristics present in continuous MEG data. SOBI, the algorithm used in this study, assumes that the components are independent across multiple time scales and tries to minimize the temporal correlation at these time scales. Of course, the assumptions made in the derivation of the blind source separation algorithm - namely statistical independence - is not $100 \%$ met in the MEG domain. However, there are theoretical reasons to believe that BSS algorithms of this sort are quite robust [3], i.e. the goodness of the separation suffers little when the assumptions are not completely met.

Therefore, it is reasonable to test whether these algorithms are actually capable of successfully separating this data in practice, just as $t$-tests are, in practice, used on non-Gaussian data due to their robustness. The physiological interpretability of our separated components is consistent with the notion that BSS algorithms are sufficiently robust in practice to obtain good performance on MEG data. Given that independent sources of noise exist within each individual neuron, and that there are regional differences in cell types, packing density, transmitter receptor distribution, and feed-forward/feed-back/lateral connectivity [10,12], it is not surprising that BSS is sufficiently robust to yield physiologically and anatomically meaningful results.

\section{Acknowledgements}

This research was supported in part by NSF CAREER award 97-02-311, the National Foundation for Functional Brain Imaging, an equipment grant from Intel corporation, the Albuquerque High Performance Computing Center, a gift from George Cowan, and a gift from the NEC Research Institute. We thank Dan Phung, Greg Kirk, and Brian Sanchez for programming support, and Michael Weisend, Robert Christner, Jeffrey Meyer, and Marianna Lanoue for their assistance in data collection.

\section{References}

[1] A.J. Bell, T.J. Sejnowski, An information-maximization approach to blind separation and blind deconvolution, Neural Comput. 7 (6) (1995) 1129-1159. 
[2] A. Belouchrani, K.A. Meraim, J.-F. Cardoso, E. Moulines, Second-order blind separation of correlated sources, In: Proceedings of the International Conference on Digital Signalling, Cyprus, 1993, pp. 346-351.

[3] J.-F. Cardoso, Blind signal separation: statistical principles, Proc. IEEE 9 (10) (1998) 2009-2025.

[4] M. Hamalainen, R. Hari, R.J. Ilmoniemi, J. Knuutila, O.V. Lounasmaa, Magnetoencephalography - theory, instrumentation, and applications to noninvasive studies of the working brain, Rev. Mod. Phys. 65 (1993) 413-497.

[5] A. Hyvarinen, Survey on independent component analysis, Neural Comput. Surv. 2 (1999) 94-128.

[6] A. Hyvarinen, E. Oja, A fast fixed-point algorithm for independent component analysis, Neural Comput. 9 (7) (1997).

[7] T.-P, Jung, C. Humphries, T.-W. Lee, M.J. McKeown, V. Iragui, S. Makeig, T.J. Sejnowski, Removing electroencephalographic artifacts by blind source separation, Psychophysiology (1999), in Press.

[8] J.D. Lewine, W.W. Orrison, II. Magnetoencephalography and magnetic source imaging, in: W.W. Orrison, II, J.D. Lewine, J.A. Sanders, M.F. Hartshorne (Eds.), Functional Brain Imaging, Mosby, St. Louis, 1995, pp. 369-417.

[9] B.A. Pearlmutter, L.C. Parra, A context-sensitive generalization of ICA, in: International Conference on Neural Information Processing, Hong Kong, September 24-27, Springer, Berlin, 1996, pp. 151-157.

[10] K.S. Rockland, Elements of cortical architecture, in: K.S. Rockland, J.H. Kaas, A. Peters (Eds.), Cerebral Cortex, Extrastriate cortex in primates, Vol. 12, Plenum Press, New York, 1997, pp. 243-293.

[11] R. Vigário, V. Jousmäki, M. Hämäläinen, R. Hari, E. Oja, Independent component analysis for identification of artifacts in magnetoencephalographic recordings, in: Advances in Neural Information Processing Systems 10, MIT Press, Cambridge, 1998.

[12] K. Zilles, S. Clarke, Architecture, connectivity, and transmitter receptors of human extrastriate visual cortex, in: K.S. Rockland, J.H. Kaas, A. Peters (Eds.), Cerebral Cortex, Extrastriate cortex in primates, Vol. 12, Plenum Press, New York, 1997, pp. 673-742. 